\title{
Editorial Risk Management: Evolution in Action?
}

\section{Denis Smith, Dominic Elliott and Alan Irwin ${ }^{1}$}

For the readership of this journal, the importance of risk as a construct within the modern world is a given rather than a matter for debate. What is however open to interpretation is the relative weight given to such factors as probability versus consequence and acute versus chronic impacts within the development of policy frameworks for the management of risk. Social responses are variable too but must be fully accounted for within the processes of risk management.

A recent visit to NewYork City has brought home the importance of these issues, if such recognition were needed, and reinforced the point that risk has become a major political, social and economic construct within the 21 st century. The manner in which the events of 11 th September (9/11) were reported in the press and the extent of the recovery work being undertaken on the crash site were powerful reminders of the scale of the disasterFlying around the US has taken on a new dimension, with large queues at check-in and frequent inspections on baggage and shoes.

For the traveller, such impositions are welcome in terms of allaying fears of further attacks, even though they come at the cost of additional travel time and some infringements of freedom. The processes of recovery and clean-up at 'ground zero' and the impact that the disaster has had on local business is evident from even a cursory exploration of the adjacent area. Indeed, the city itself has a subdued air about it that is unavoidably associated with the aftermath of the disaster The disaster has pointed to the difficulties that can face organisations which had not previously considered themselves at risk from such catastrophic events, and highlights the dif ficulties associated with developing effective contingency plans for (thankfully) rare events.The concept of risk has clearly taken on a new dynamic in the wake of $9 / 1$, although one cannot help feeling that organisations, and more importantly governments, may well fail to learn lessons in the long term.

Many commentators have observed that the events of $9 / 11$ will forever shape our perceptions of risk. In that way, the disaster will serve as a defining moment in the history of the 21 st century. Technically, that century began before the Twin Towers catastrophe took place. However, economic, social, political and cultural trends rarely fit the formal time frames developed by humankind; instead, we tend to see the passage of time in terms of defining momentS.his notion of a defining moment raises some interesting issues for this journal, some of which can be briefly developed here in the hope of stimulating further debate. Of particular importance are the notions of what makes a defining moment, what is the role of space and time in risk events, and to what extent we can anticipate as well as respond to such tragedies.

Exploring the notion of a defining moment is itself important when we are discussing issues relating to risk. For many historians the 20th century proper began on 28th June 1914 with the assassination of Arch-Duke Ferdinand. This event was to serve as a trigger for the catastrophe that followed, and exposed the inherent weakness within the 'system' represented by the politics of Western Europe at that point in space and time. Similarly, the sinking of the Titanic has been hailed as a defining moment in the management of technology . The manner in which this 
supposedly unsinkable ship could founder with the loss of so many passengers was a shock event for Western society in the early part of the 20th century, where there was a growing belief in the power of technology and our ability to manage it successfully. The fact that managers appeared to be so complacent about the risk of the ship foundering led to a lack of adequate lifeboat provision, no lifeboat drills and an apparent disregard for the risks of iceThe Titanic has become a metaphor for risk-taking on the part of management and for the manner in which ganisations can become complacent about their strategies for dealing with such risks.

What sort of metaphor will the disaster of $9 / 1 \quad 1$ become over time? This raises a number of questions concerning the manner in which events have an important defining quality and the extent to which risk is a constituent element within this. Is the event 'defining' because it shatters the previously held paradigms on relative risk, or because it shows that the precautionary measures put into place by managers were limited? Can it also be considered as defining because it illustrates the limitations of the notion of a worst-case scenario in planning for adverse events? In all cases, this notion of a defining event is influenced to a significant degree by the manner in which the event is grounded within both space and time.

Notions of time and space have proved themselves to be important constructs within the literature on post-modern perspectives on societal problems. Within discussions on risk, space and time are also important. At a simple level, concepts of space influence the manner in which the range of the impacts associated with the event are felt. This concept of hazard range has proved to be important in shaping the response of oganisations to the adverse event itself.The spatial dynamics of risk can be seen in terms of disease patterns (as illustrated by the 2001 foot and mouth outbreak in the UK) and also in the interconnected nature of risk events, as illustrated by thel th September attacks.

Time is also an important factor within debates on risk. Notions of immediate consequences versus those whose effects are delayed have been evident in much of the debates surrounding environmental management over the last 30 years. The current concerns about mobile phones and the MMR vaccine, and the ever-present debates about global warning and ozone depletion, illustrate the manner in which concerns about the temporal dynamics of risk shape policy and political debate. The interaction between space and time also impacts upon the tightly-connected character of our systems. The manner in which failure modes create events that are rapid in their emergence and complex in terms of the interaction of causal factors has beguiled many attempts to manage complex, non-linear systems.

Finally, problems associated with organisational culture and management have also been at the forefront of the public consciousness in terms of risk. Events involving Railtrack in the UK and the subsequent resignation of the chief executive may have heralded a new era in terms of accountability and risk. That said, there are many who feel that such moves are inadequate to ensure that corporations continue to prioritise notions of hazard over other performance measures within the organisation. The collapse of Enron in the US has raised further questions about the ethical aspects of management. Clearly, there is a range of issues that emege from the behaviour of managers and the process of management, and these will remain at the forefront of research within the field. Such issues include: the manner in which managers conceptualise notions of risk and determine its acceptability; the nature of the communication process around risk; the articulation of complex, ill-defined processes; and the trade-offs that occur between short-term gain and long-term consequences. These are not new issues for risk management but they remain problematic for organisations and groups within society alike.

Against the background of such issues, this journal has continued to evolve and is attempting to move into a new phase in its development. Our central aim is that the journal should provide a 
focus for the latest research across the broad area that is the field of risk. It should provide a robust forum for debate and discussion around the key constructs of risk and crisis, and shouldultimately - seek to become the first choice of professionals publishing in this field. The development of the editorial and advisory boards has been undertaken with a view to encompassing as much of this diverse field as possible. It is our hope that the journal will continue along its evolutionary path, with a view to ensuring that issues of risk management become more firmly embedded within both corporate and social consciousness.Along the way, it is our firm intention that we should not only be able to improve our understanding of risk events but also establish a more transparent and robust process for minimising their future occurrence and impact.

\section{Notes}

1 Denis Smith is in the University of Liverpool Management School, Dominic Elliott in the Sheifld University Management School andAlan Irwin in the School of Social Sciences, Brunel University 